\title{
A novel tool for stable genomic reporter gene integration to analyze heterogeneity in Photorhabdus luminescens at the single-cell level
}

\author{
Angela Glaeser and Ralf Heermann \\ BioTechniques 58:74-81 (August 2015) doi 10.2144/000114317 \\ Keywords: phenotypic heterogeneity; promoter activity; genomic integration; Photorhabdus \\ Supplementary material for this article is available at www.BioTechniques.com/article/114317.
}

Ludwig-Maximilians-Universität München, Biozentrum, Bereich Mikrobiologie, Martinsried/München, Germany

Determination of reporter gene activity at the single-cell level is a prerequisite for analyzing heterogeneous gene expression in bacteria. The insect pathogenic enteric bacterium Photorhabdus luminescens is an excellent organism in which to study heterogeneity since it exists in two phenotypically different forms, called the primary and secondary variant. A tool for generating stable genomic integrations of reporter genes has been lacking for these bacteria, and this has hampered the acquisition of reliable data sets for promoter activities at the single-cell level. We therefore generated a plasmid tool named pPINT-mCherry for the easy and stable introduction of gene fragments upstream of an mCherry reporter gene followed by stable integration of the plasmid into the $P$. luminescens genome at the rpmE/g/mS intergenic region. We demonstrate that the genomic integration of reporter genes for single-cell analysis is necessary in $P$. luminescens since plasmid-borne reporter genes mimic heterogeneity and are therefore not applicable in these bacteria, in contrast to their use in single-cell analysis in other bacteria like Escherichia coli.

It is well recognized that genetically identical bacteria often show a high degree of variability in homogenous environments, an observation that is referred to as phenotypic heterogeneity (1). Photorhabdus luminescens is an enteric bacterium that lives in mutualistic association with soil nematodes and is highly pathogenic for insects (2). The bacteria are of high biotechnological and pharmaceutical interest since they are already used as bio-insecticides in association with their nematode host (3), and they are also potentially a rich reservoir of as yet unknown natural compounds (4). $P$. luminescens exists in two pheno- typically different forms, designated as the primary and secondary variant. Single primary cells undergo phenotypic switching and convert to secondary cells after prolonged cultivation. Primary cells exhibit phenotypic characteristics, such as the production of several extracellular enzymes, pigments, antibiotics, bioluminescence, and crystalline inclusion proteins, that are absent from the secondary variant $(5,6)$. While both variants are equally pathogenic to insects, secondary cells are unable to support nematode growth and development (5). For these reasons, P. luminescens is an excellent model organism for the study of molecular mechanisms of bacterial phenotypic heterogeneity. Here we describe a novel tool for the stable integration of promoterreporter genes into the $P$. luminescens genome that allows for analysis of heterogeneous gene expression at the single-cell level. Our results indicate that plasmid-borne tools are not suitable for analysis of heterogenous gene expression in these bacteria.

\section{Materials and methods}

Materials

Strains used in this study are listed in Supplementary Table S1, plasmids are

\section{METHOD SUMMARY}

The plasmid pPINT-mCherry is an easy-to-use tool for the stable integration of reporter genes at the rpmE/glmS site into the Photorhabdus luminescens genome. A specific promoter region can be cloned into the multiple cloning site (MCS) upstream of the mCherry gene and, after conjugation of the plasmid into $P$. luminescens, pPINT-mCherry integrates via homologous recombination at the specific single site into the genome. Correct clones can be positively selected due to the conferred antibiotic resistance. If required, the plasmid backbone and therefore the antibiotic resistance can be removed from the chromosome by the use of sacB negative selection. 
A

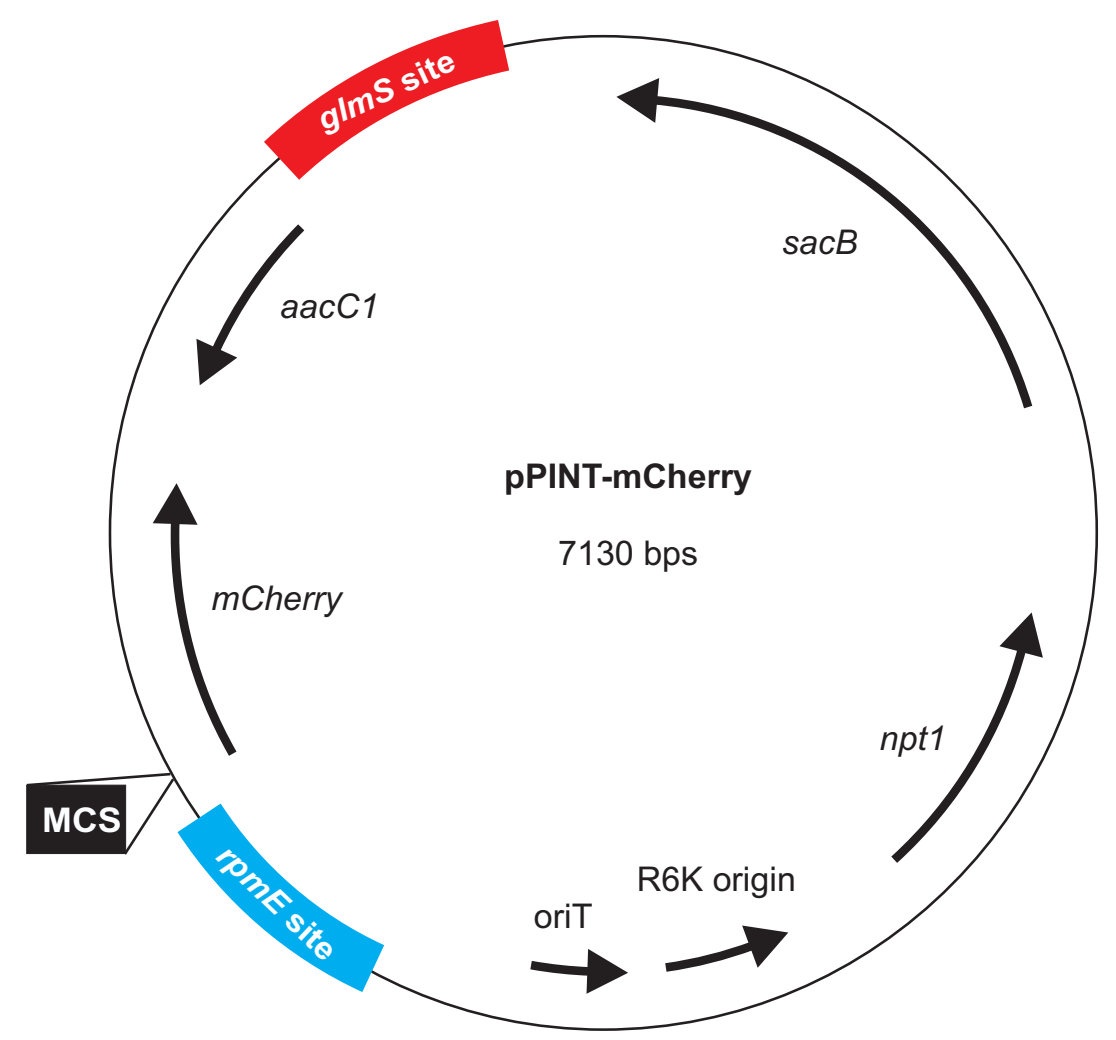

B

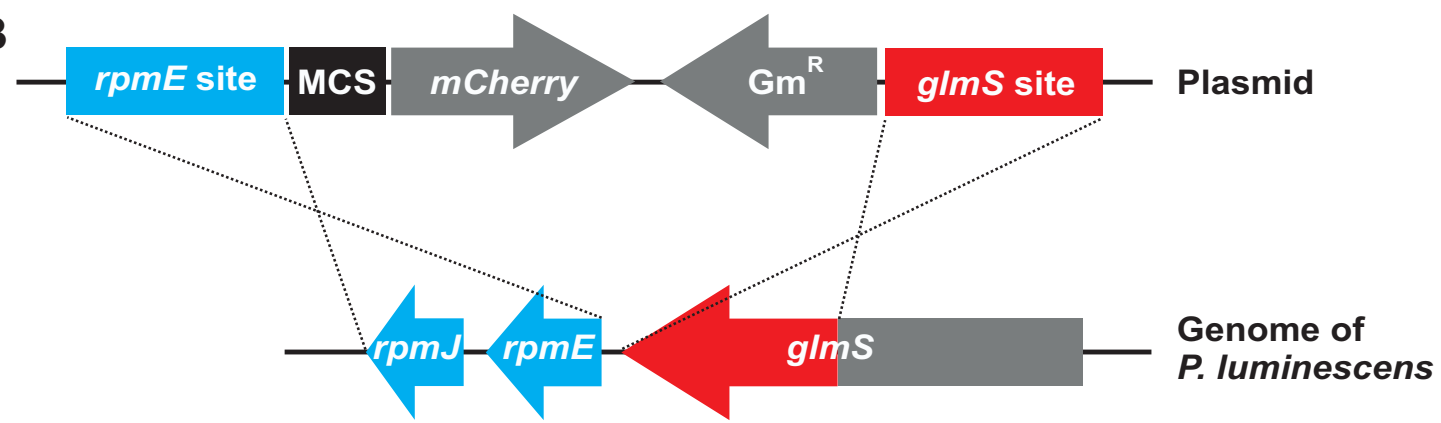

Figure 1. The mobilizable and integrative vector pPINT-mCherry. (A) Vector map. The plasmid carries kanamycin (npt1) and gentamicin (aacC1) resistance cassettes. pPINT-mCherry integrates into the P. Iuminescens genome at the rpmE/g/mS site via the two 600-bp long homologous flanking regions. Transcriptional as well as translational reporter genes can be generated by inserting the respective DNA into the multiple cloning site (MCS) upstream of $m$ Cherry. pPINT-mCherry carries the R6K origin so that it only replicates in strains carrying the $\lambda$ pir gene. The vector is mobilizable due to the presence of the origin of transfer oriT. The negative selection marker sacB allows vector backbone removal after insertion into the chromosome. (B) Homologous sites in pPINT-mCherry used for recombination into the intergenic region of the rpmE and g/mS genes in $P$. luminescens.

listed in Supplementary Table S2, and primers are listed in Supplementary Table S3. PCR was performed using the Q5 Polymerase or OneTaq Polymerase from New England Biolabs (Frankfurt, Germany). Restriction enzymes and T4 DNA ligase were also purchased from New England Biolabs. Plasmid isolations were performed with the HiYield Plasmid Mini Kit and the HiYield PCR DNA Fragment Extraction Kit was used for purification of PCR products
(Süd-Laborbedarf, Gauting, Germany). Genomic DNA was prepared using the Ultra-Clean Microbial DNA Isolation Kit (Mo Bio Laboratories Inc., Carlsbad, $\mathrm{CA})$.

Bacterial strains and growth conditions $P$. luminescens and $E$. coli were aerobically grown in LB medium $[10 \%(\mathrm{w} / \mathrm{v})$ peptone, $5 \%(\mathrm{w} / \mathrm{v})$ yeast extract, $10 \%$ $(\mathrm{w} / \mathrm{v}) \mathrm{NaCl}]$ at $30^{\circ} \mathrm{C}$ (P. luminescens) and $37^{\circ} \mathrm{C}$ (E. coli). For preparation of agar plates, $1.5 \%(\mathrm{w} / \mathrm{v})$ agar was added to the medium. If necessary, media were supplemented with kanamycin $(50 \mu \mathrm{g} /$ $\mathrm{mL})$, ampicillin $(100 \mu \mathrm{g} / \mathrm{mL})$, gentamicin $(15 \mu \mathrm{g} / \mathrm{mL})$, and/or rifampicin $(50 \mu \mathrm{g} / \mathrm{mL})$. To allow the growth of the donor strain E. coli ST18, the medium was supplemented with 5-aminolevulinic acid (50 $\mu \mathrm{g} / \mathrm{mL}$ ) (7). Pre-cultures were grown overnight, and the following day, appropriate volumes of the pre-cultures were used for inoculation of the main cultures 
A

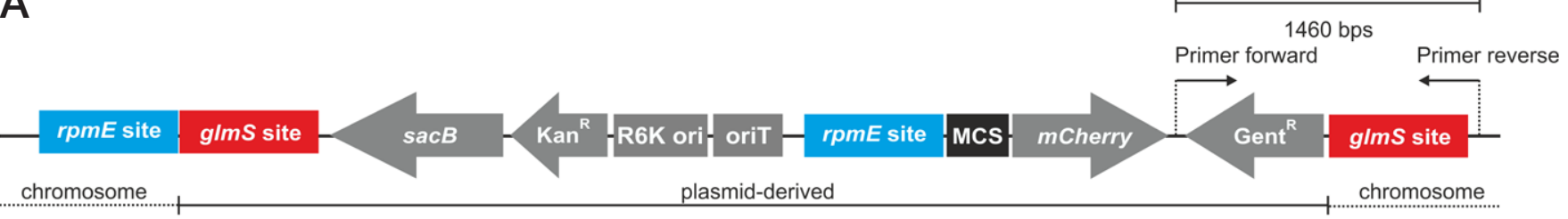

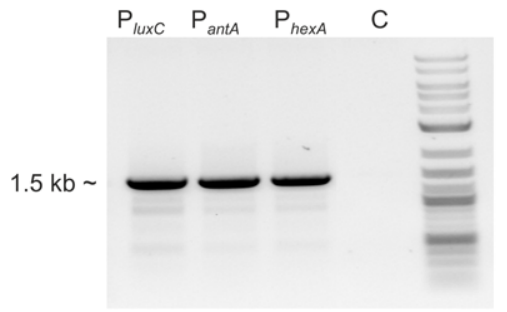

$\mathrm{P}_{\text {luxc }}:$ TT01 rpmE/ glmS site:: pPINT-P luxc -mCherry

$\mathrm{P}_{\text {antA }}$ : TT01 rpmE/ glmS site:: pPINT-P $\mathrm{P}_{\text {ant }}$-mCherry

$\mathrm{P}_{\text {hexA }}:$ TT01 rpmE/ glmS site:: pPINT-P $\mathrm{P}_{\text {hexA }}-m$ Cherry

C: $\quad$ TT01 wild type
B

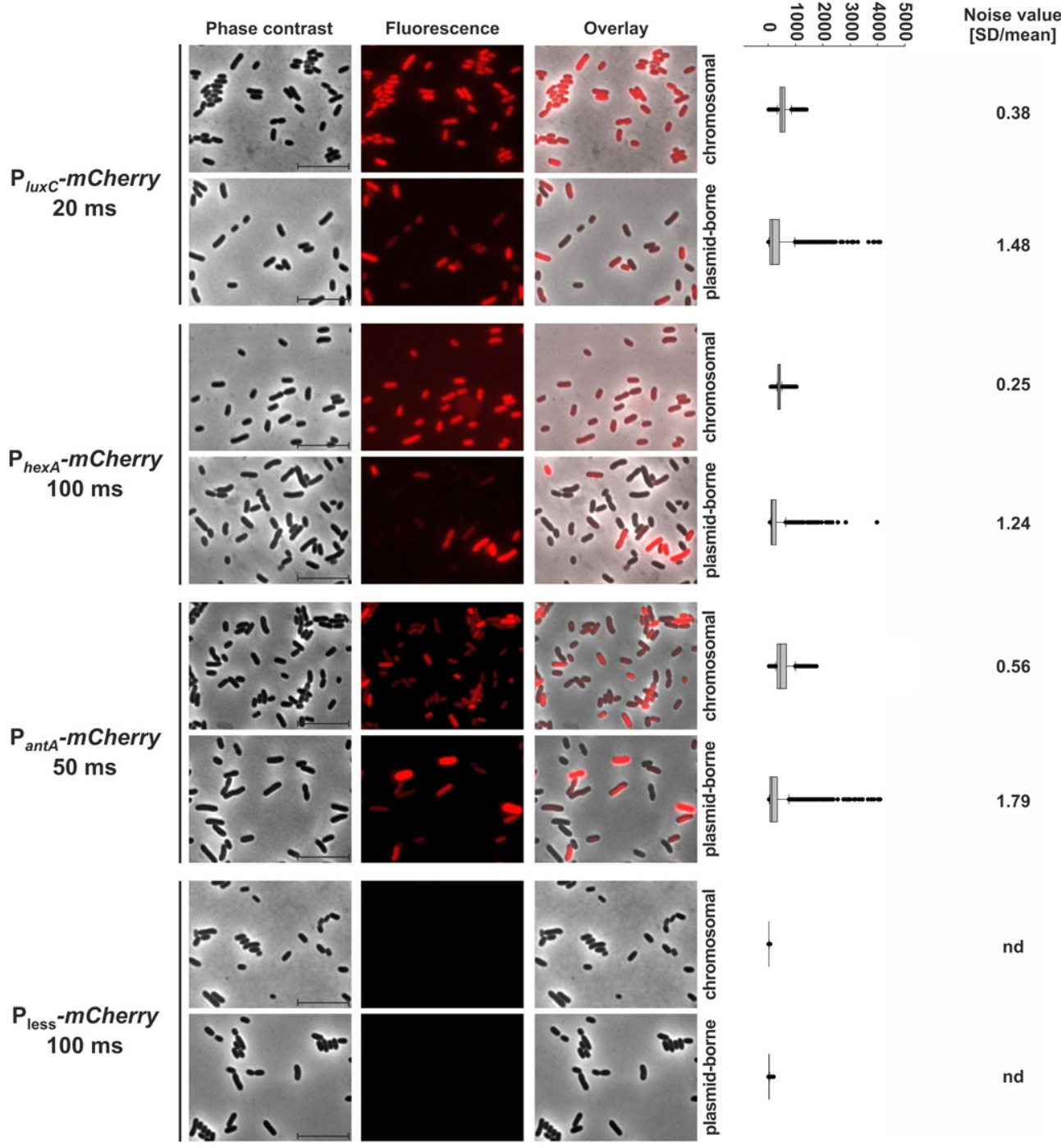

Figure 2. Generation and single-cell microscopy imaging of $P$. luminescens $P_{\text {uxc }}-m$ Cherry, $P$. luminescens $P_{\text {anta }}-m$ Cherry, and $P$. luminescens $\mathbf{P}_{\text {hexA }}$ - $m$ Cherry. (A) Generation of the reporter strains. The first recombination step to integrate the reporter genes into the $P$. luminescens genome was performed by conjugation via the E. coliST18 donor carrying the respective pPINT-mCherry plasmids. The correct insertions of the respective pPINT-mCherry derivatives into the $P$. luminescens chromosome were verified by PCR with one primer annealing in the reporter gene region of the plasmid (primer forward) and one primer annealing downstream of the integrated vector in the genome (primer reverse), using genomic DNA as template. When the integration occurred via the rpmE site, the correct insertion of the respective pPINT-mCherry derivative resulted in the amplification of a 1460-bp DNA fragment. C: Control using genomic DNA of wild-type $P$. luminescens. (B) Single-cell microscopy imaging of plasmid-borne and chromosomally integrated promoter-mCherry fusions in $P$. luminescens. $P$. luminescens $\mathrm{P}_{\text {luxc }}-m$ Cherry, $P$. luminescens $P_{10+4}-m$ Cherry, $P$. luminescens $\mathrm{P}_{\text {hexA }}-$ mCherry, and $P$. luminescens $P_{\text {less }}-m$ Cherry (control) harboring the respective reporter genes in the chromosome using homologous recombination via pPINT-mCherry, as well as $P$. luminescens $\mathrm{pBR}-\mathrm{Km}$ $P$ - $m$ Cherry, $P$. luminescens $\mathrm{pBR}-$ $\mathrm{Km}-P_{\text {anta }}-\mathrm{mCherry,} P$. luminescens pBR-Km-P $\mathrm{P}^{\text {and }}$-mCherry, and P. Iuminescens $\mathrm{pBR}-\mathrm{Km}-\mathrm{P}_{\text {less }}$-mCherry (control) were cultivated for $24 \mathrm{~h}$ in the presence of the appropriate antibiotics. The cells were then analyzed for promoter activities at the singlecell level by fluorescence microscopy. The exposure time is given in ms and was adjusted for each promoter depending on its strength. On the right panel the corresponding boxplots are shown. The fluorescence intensities of 500 single cells were individually measured for each experiment, resulting in 1500 cells of the respective triplicates being analyzed using the software Big Cell Brother (11). The noise values were calculated as SD/mean from 1500 cells. Representative images from one of three independently performed experiments are shown. 
to an $\mathrm{OD}_{600}$ of 0.05 . For measurement of the fluorescence intensities of the $\mathrm{P}_{\text {lac }}$-eyfp fusions, $0.5 \mathrm{mM}$ IPTG was added to activate the lac promoter.

\section{Plasmid generation}

For the generation of plasmid pPINT, the rpmE and g/mS homologous regions of the $P$. luminescens genome were amplified via PCR. The rpmE site containing the genes rpmE and rpmJ was amplified using primers FA_ rpmE-Spel_fwd and FA_rpmE-mcs_rev with genomic DNA of $P$. luminescens TT01 as template. The PCR product of the glms site, containing a part of the glmS gene, was amplified using primers FB_glmS-mcs_fwd and FB_ glmS-Sall_rev with genomic DNA of $P$. luminescens as template. Subsequently, an overlap PCR of the rpmE and the $\mathrm{g} / \mathrm{mS}$ site as templates was performed using primers FA_rpmE-Spel_fwd and FB_glmS-Sall_rev. The overlap PCR product was inserted into the plasmid pNPTS-138-R6KT (8) via the restriction enzymes Spel and Sall, resulting in the plasmid PPINT. A fragment containing a multiple cloning site, mCherry [template: pBR-mCherry (9)], and a gentamicin resistance cassette (template: pUC18R6KT-mini-Tn7T-Gm) was generated via PCR using the primer pairs cherry-mcs-pNPTS_fwd/cherrymcs-pNPTS_rev and gmR-pNPTS_fwd/ gmR-pNPTS_rev, respectively, followed by an overlap PCR. The overlap PCR fragment was inserted via EcoRl and Eagl sites into the plasmid pPINT, resulting in the plasmid pPINT-mCherry.

For generation of plasmid pBR-KmmCherry the kanamycin cassette [template: pNPTS-138-R6KT] was amplified via PCR using the primers $\mathrm{Km}$-Pstl_fwd and Km-EcoRI_rev. The resulting fragment was cut with the restriction enzymes Pst and EcoRI and ligated with the equally treated plasmid pBR-Cherry. In order to create the plasmid $\mathrm{pBR} / \mathrm{Km}-\mathrm{P}_{\text {lac- }}$ eyfp/ $\mathrm{P}_{\text {hexA- }}$ mCherry, $\mathrm{P}_{\text {lac }}$-eyfp was amplified using primers Plac-eyfp-Dral_fwd and eyfp-Dral_rev with plasmid pEYFP (Takara-Clonetech, Saint-Germain-enLaye, France) as template. The PCR product was inserted into the plasmid pBR-Cherry-Km-P $P_{\text {hexA }}$ via the Dral restriction site. Since $P$. luminescens has no Lacl repressor, the promoter of the lacZYA operon could be used as a constitutively activated promoter without adding inducer. The promoters $P_{\text {ant } A}, P_{\text {hexA }}$, and $P_{\text {luxc }}$ were amplified by PCR with the primers PantA-BamHI_ fwd/PantA-Xmal_rev, PhexA-BamHI_ fwd/PhexA-Xmal_rev, and PluxCBamHI_fwd/PluxC-Xmal_rev using $P$. luminescens genomic DNA as template. The PCR products were cloned into plasmid pPINT-mCherry or pBR-KmmCherry using the restriction sites BamHI and Xmal. Restriction analysis as well as DNA sequencing (Genomics core facility, LMU Biozentrum) verified the accuracy of all generated plasmids.

Competent cells and transformations E. coli cells were made chemically competent and transformed as described elsewhere (10). P. luminescens was made electrocompetent and transformed by electroporation. P. luminescens cells were cultivated in $\mathrm{LB}$ medium at $30^{\circ} \mathrm{C}$ up to an $\mathrm{OD}_{600}$ of 0.8-1.2. The cells were harvested by centrifugation at $18^{\circ} \mathrm{C}$, and the cell pellet was resuspended in the same volume of $10 \%$ (v/v) glycerol and centrifuged again. Cells were then washed with $1 / 2$ of the starting volume in 10\% (v/v) glycerol and, after centrifugation, were washed again with 1/10 of the starting volume. After another centrifugation step, the cells were resuspended with 1/250 of the starting volume in 10\% (v/v) glycerol. Sixty microliters of cell suspension was mixed with 100-200 ng plasmid DNA and transferred into $0.2 \mathrm{~cm}$ electroporation cuvettes. Electroporation was performed with a pulse of $2500 \mathrm{~V}$ for 4-6 ms. Cells were then mixed with 1 $\mathrm{mL}$ LB medium and incubated aerobically at $30^{\circ} \mathrm{C}$ for 1 hour. The cells were centrifuged and resuspended in $100 \mu \mathrm{L}$ LB. The samples were then spread on appropriate agar plates and incubated at $30^{\circ} \mathrm{C}$ for 2 days.

Integration of reporter genes into the $P$. luminescens genome Integration of promoter-mCherry reporter genes into $P$. luminescens was performed via homologous recombination. The conjugative plasmid transfer from donor strain E. coli ST18 containing the required plasmid into $P$. Iuminescens TT01 was performed by the filter mating method (7). First, the donor and

Introducing KAPA HYPER PLUS

Single-tube DNA fragmentation and library preparation in 2.5 hours

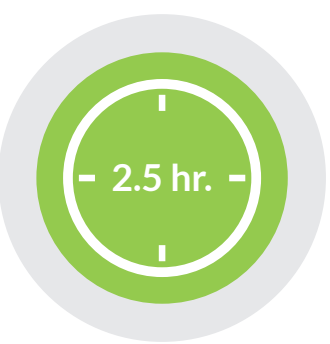

Speed of tagmentation

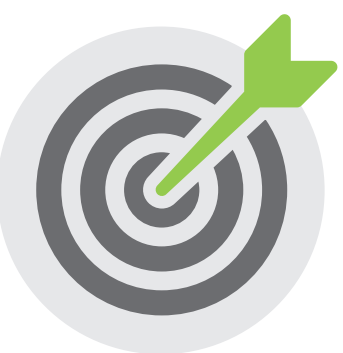

Quality of mechanical shearing

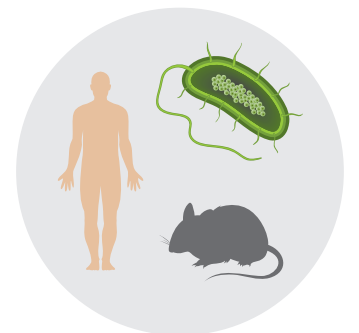

Flexible sample types and input amounts

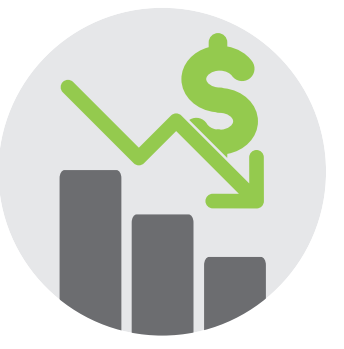

Reduced sequencing costs

\section{Visit}


B
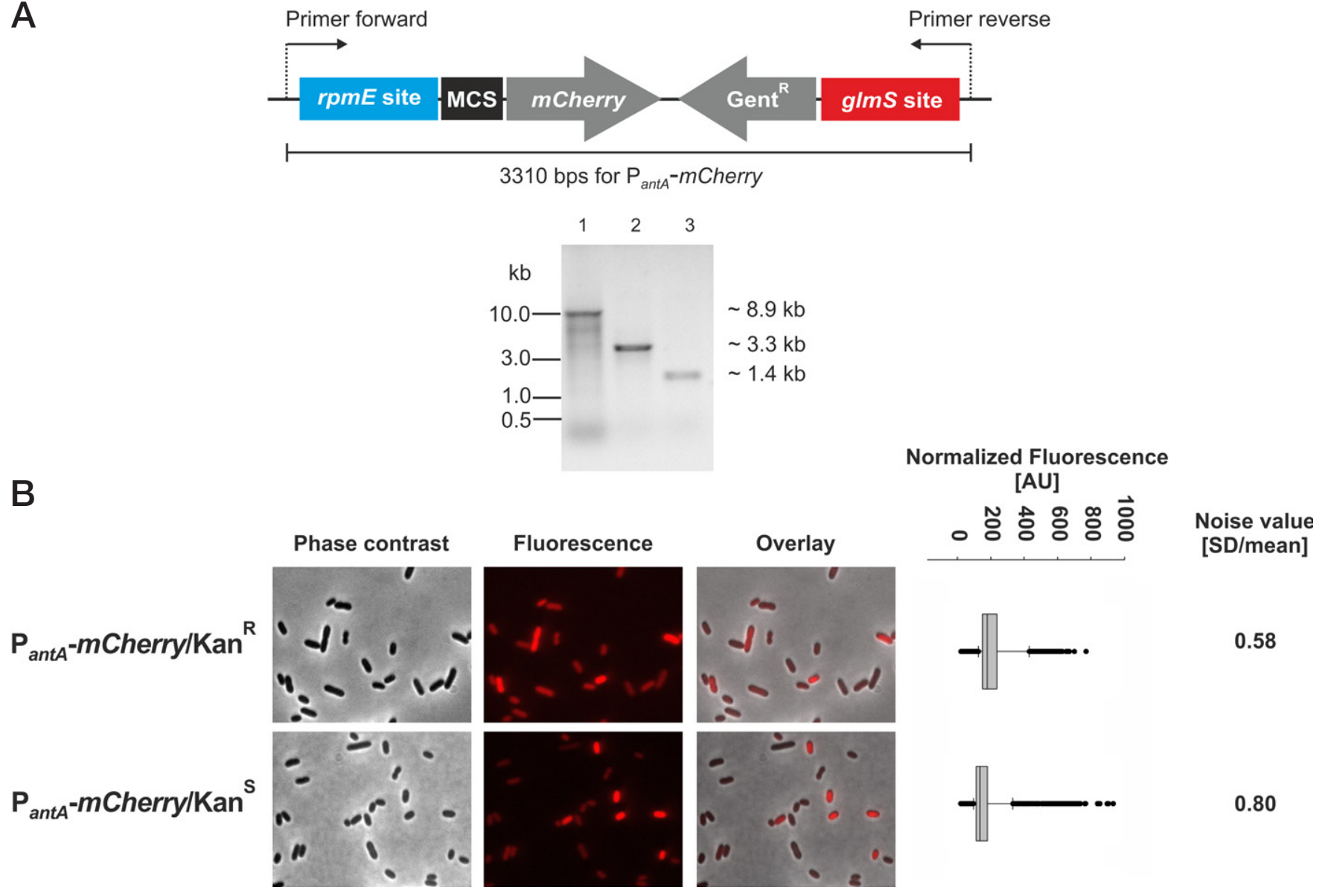

Primer reverse

Figure 3. Influence of pPINT-mCherry plasmid backbone on reporter gene fluorescence intensity and heterogeneity. (A) Removal of the vector backbone in strain $P$. Iuminescens $\mathrm{P}_{\text {ants }}-m$ Cherry. The pPINT-mCherry vector backbone was removed by a second homologous recombination and selection of Kan ${ }^{\varsigma}$, Gm ${ }^{R}$, and Suc ${ }^{R}$ clones. The resulting strain was named $P$. luminescens $\mathrm{P}_{\text {luxc }}-m$ Cherry-Kans. The removal of the backbone was checked via PCR using one primer binding upstream (primer forward) and one primer binding downstream (primer reverse) of the rpmE/g/mS site. The PCR product for the first recombination of pPINT-P $P_{\text {anta }}$-mCherry resulted in a band with a size of $8863 \mathrm{bp}$; for the second homologous recombination (loss of plasmid backbone), the DNA fragment was $3310 \mathrm{bp}$. No insertion at the rpmE/g/mS site resulted in amplification of a DNA fragment with $1358 \mathrm{bp}$. Genomic DNA was used as template: Lane 1, P. Iuminescens $\mathrm{P}_{\text {luxc }}-m$ Cherry/Kan ${ }^{R}$; Lane 2, P. luminescens $\mathrm{P}_{\text {luxc }}-m$ Cherry/Kans; and Lane 3, P. Iuminescens wild-type (control). (B) Single-cell microscopy imaging of $P$. luminescens $\mathrm{P}_{\text {ants }}-m$ Cherry/Kan ${ }^{R}$ and $P$. luminescens $\mathrm{P}_{\text {anta }}-m$ Cherry/Kan ${ }^{S}$. P. luminescens $\mathrm{P}_{\text {anta }}-m C h e r r y / K^{2} n^{R}$ (remaining pPINT-mCherry plasmid backbone) and $P$. luminescens $\mathrm{P}_{\text {ants }}-m$ Cherry/Kan ${ }^{\mathrm{S}}$ (pPINT-mCherry plasmid backbone removed) were cultivated and analyzed for reporter gene activity by fluorescence microscopy. On the right panel, the corresponding boxplots are shown. The fluorescence intensities of 500 single cells were individually measured for each experiment, resulting in 1500 cells from the respective triplicates being analyzed using the software Big Cell Brother (11). The noise values were calculated as SD/mean from 1500 cells. Figures each represent one characteristic result out of three independently performed experiments.

the recipient strains were cultivated in LB medium, supplemented with the respective additives if required, up to an $\mathrm{OD}_{600}$ of $0.8-1.0$, The donor strain was washed 3 times in LB medium and mixed with the recipient strain in a ratio of $1: 5$ in a final volume $1 / 10$ of the donor's initial culture volume. The two strains were pooled and dropped onto a nitrocellulose filter located on a LB agar plate. After incubation overnight at $30^{\circ} \mathrm{C}$, the cells were resuspended in $500 \mu \mathrm{L}$ LB, spread onto LB agar plates containing the required antibiotics, and incubated for 2 days at $30^{\circ} \mathrm{C}$. Single colonies were checked for chromo- somal integration of the plasmid via PCR (check-rpmE_fwd, oriT_fwd, gmR-pNPTS_fwd, check-mcherryins_rev, check-glmS_rev) using the genomic DNA of the clones as template, and subsequent DNA sequencing of the DNA fragments was performed. For removal of the vector backbone in strain $P$. luminescens $P_{\text {anta }}$-mCherry, the respective strain was grown for $24 \mathrm{~h}$ in LB medium containing gentamicin in the absence of kanamycin, and then streaked on LB agar containing 10\% (w/v) sucrose and gentamicin. Clones were checked for kanamycin sensitivity by streaking on LB agar containing kanamycin. The overall efficiency of vector backbone loss was determined to be $>95 \%$, when $50-100$ individual clones were checked. The resulting strain was named $P$. luminescens $P_{\text {ant }}{ }^{-}$ $m$ Cherry/Kans Genomic DNA of Kans ${ }^{s}$, $\mathrm{Suc}^{R}$, and $\mathrm{Gent}^{\mathrm{R}}$ clones was isolated and correct integration was verified by PCR using primers check-rpmE_fwd and check-glmS_rev annealing outside the homologous regions, and subsequent DNA sequencing was performed.

Promoter activity analysis

Promoter activities of $P$. luminescens reporter strains were investigated via 
mCherry and eYFP fusions with a fluorescence microscope (Leica, Bensheim, Germany). An excitation wavelength of $546 \mathrm{~nm}$ and a $605 \mathrm{~nm}$ suppression filter with $75 \mathrm{~nm}$ bandwith was used to measure mCherry. For measuring eYFP, an excitation wavelength of $460 \mathrm{~nm}$ and a $512 \mathrm{~nm}$ suppression filter with $75 \mathrm{~nm}$ bandwith was used. The main cultures were inoculated at an $\mathrm{OD}_{600}$ of 0.05 with the appropriate volume of the respective pre-cultures and were grown at $30^{\circ} \mathrm{C}$ for $24 \mathrm{~h}$ in the presence of appropriate antibiotics. Then, $7 \mu \mathrm{L}$ of the cultures were dropped on an agarose pad $[0.5 \%(w / v)$ agarose in PBS buffer, $\mathrm{pH}$ 7.4] located on a microscope slide and covered with a coverslip. Three independent biological experiments were performed. For each experiment, the fluorescence intensities of 500 cells were measured using the single-cell analysis tool Big Cell Brother (11).

\section{Results and discussion}

To analyze heterogeneity of gene expression at the single-cell level, a molecular tool is necessary for the stable and unique integration of reporter genes into the chromosome, but this has not yet been available for P. luminescens. In many Gram-negative bacteria, genomic integrations can in principle be achieved using the mini transposon $\mathrm{Tn} 7$, which has a unique attachment site located in the rpmE/g/mS intergenic region (12). Although this attachment site is also present within the $P$. luminescens genome, located between the genes rpmE (p/u0036) and $\mathrm{g} / \mathrm{mS}$ (p/u0037), stable mini-Tn7 integration can be challenging. The $P$. luminescens genome appears to have multiple specific or nonspecific Tn7-attachment sites, since integration of mini-Tn7 derivatives often occurs elsewhere beyond the rpmE/g/mS intergenic region in this organism (unpublished observations). To surmount this problem, we generated the mobilizable plasmid pPINT-mCherry (Figure 1A), which can specifically integrate via homologous recombination at the rpmE $/ \mathrm{g} / \mathrm{mS}$ site of $P$. luminescens (Figure 1B). Two other known Photorhabdus species, $P$. temperata and $P$. asymbiotica, also contain an rpmE/glmS intergenic region sharing $87 \%$ and $80 \%$ identity with the P. luminescens rpmE/g/mS site, respectively. Since the $\mathrm{g} / \mathrm{mS}$ and rpmE sequences in pPINT-mCherry can be replaced using the Spel/Pst and Eagl/Sall restriction sites, respectively, pPINT-mCherry could easily be modified for functionality in other species than P. luminescens. The plasmid pPINTmCherry carries the mCherry gene with an upstream multiple cloning site (MCS) to allow the easy insertion of promoters and/or gene fusions. pPINTmCherry derives from the plasmid pNPTS138-R6KT (8), which contains the R6K origin of replication and thus does not replicate in $P$. luminescens. Since pPINT-mCherry is mobilizable, it can be transferred into $P$. luminescens via conjugation using the $E$. coli strain ST18 as the donor (7) and then easily be out-selected due to 5-aminolevulinic acid auxotrophy. We determined the pPINT-mCherry conjugation/recombination efficiency as $1.15 \pm 0.14 \times 10^{-6}$. Furthermore, pPINT-mCherry contains the sacB gene encoding the levansucrase of Bacillus subtilis, allowing removal of the vector backbone from the chromosome by growing the cells in the presence of sucrose (13). For positive selection, we also inserted a gentamicin resistance cassette between the recombination sites to facilitate screening after excision of the vector backbone.

We used pPINT-mCherry for the stable integration of different promoterreporter genes into the $P$. luminescens genome. We chose promoters from three different $P$. luminescens genes: (i) the bioluminescence luxCDABE operon, since bioluminescence is a characteristic feature of $P$. luminescens; (ii) the regulatory gene hex $A$ as an example of a gene encoding a transcriptional regulator; and (iii) the anthraquinone synthesis operon antABCDEFGHI, a gene cluster responsible for secondary metabolite synthesis. As the first step, we generated $\mathrm{P}_{\text {luxC }}-m$ Cherry, $\mathrm{P}_{\text {hexA }}$ $m$ Cherry, and $\mathrm{P}_{\text {ant }}$ - $m$ Cherry fusions

\section{Goodbye thermocycler! \\ Revolutionary DNA Detection}

TwistDx

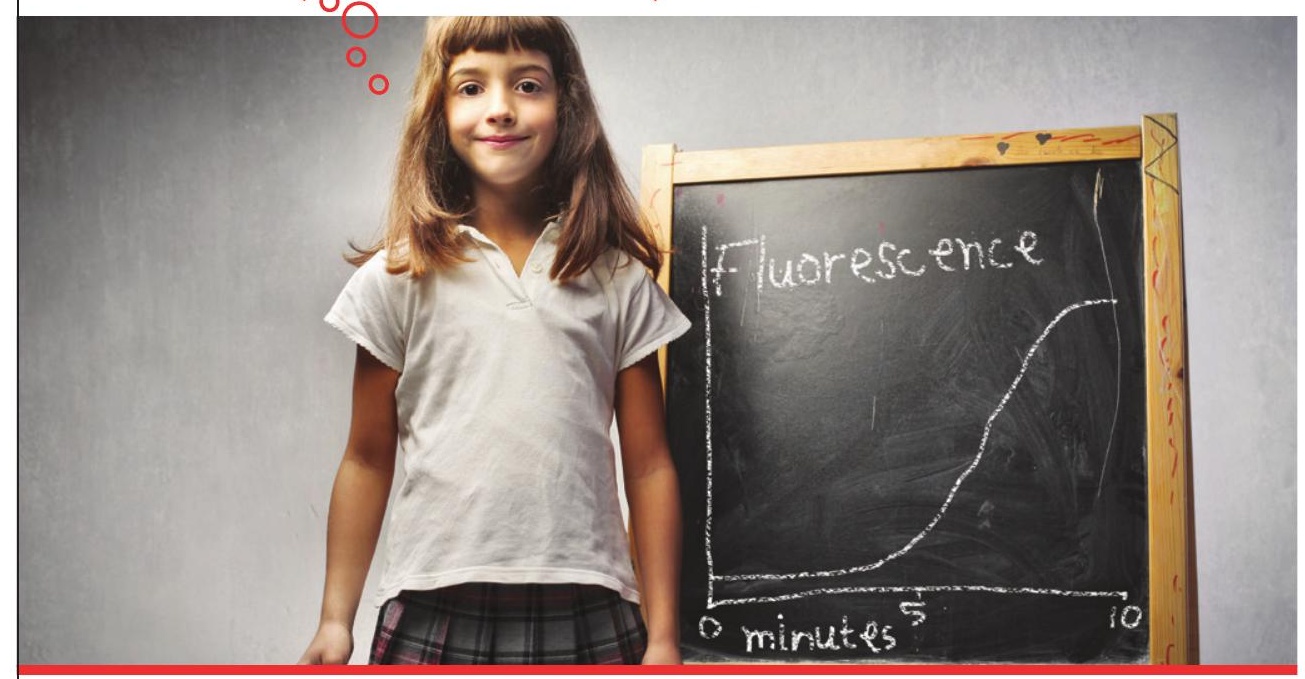

Enjoy more playtime with Recombinase Polymerase Amplification (RPA) the isothermal amplification that really works. RPA uses a recombinase-based process instead of thermocycling to amplify DNA, meaning real-time detection within 15 minutes.

Read over 50 inspirational RPA publications twistdx.co.uk/publications
RPA. It really works. twistdx.co.uk | +44 (0)1223 496700 

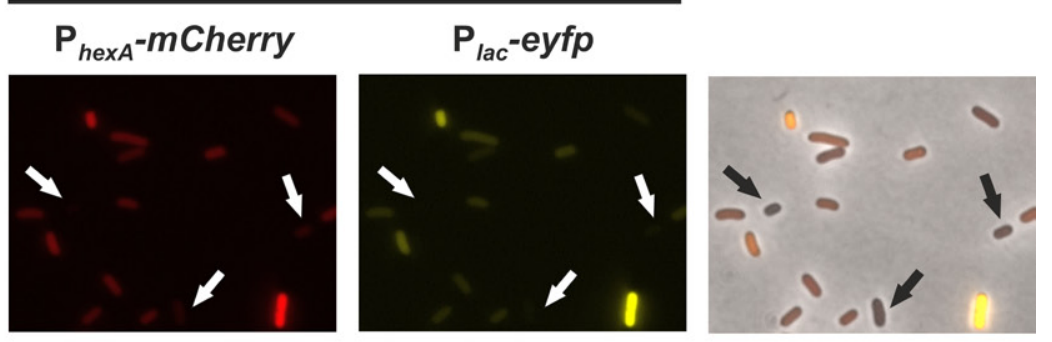

\section{E. coli}
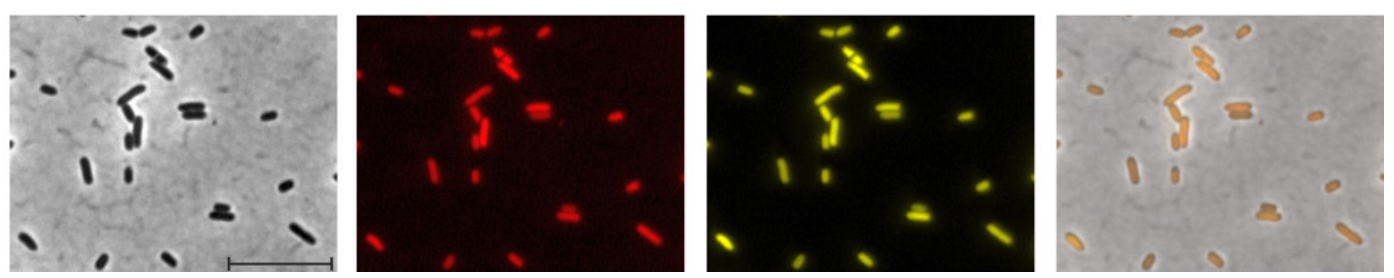

Figure 4. Single-cell microscopy imaging of $P$. Iuminescens and $E$. coli reporter strains carrying two different reporter genes encoded on one

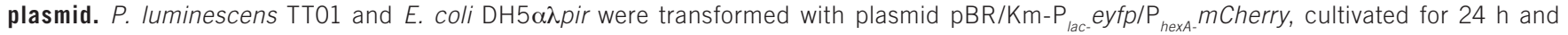
analyzed for promoter activities at the single-cell level by fluorescence microscopy (red and yellow channels). The promoter of hexA was fused to $m C$ herry, and the promoter of lacZYA was fused to eyfp. The arrows highlight cells that are not fluorescent at all. Figures each represent one characteristic result out of three independently performed experiments.

by cloning at least $350 \mathrm{bp}$ upstream of the start codon of the respective genes into the pPINT-mCherry plasmid and then integrated these three reporters into the genome of $P$. luminescens primary cells via conjugation followed by homologous recombination. The proper insertion of the reporter genes at the rpmE/g/mS intergenic region could be verified in all of the clones tested (Figure 2A). Subsequently, we compared the fluorescence intensities of the mCherry reporter strains derived either from the plasmid pBR-mCherry (9), which is a low-copy plasmid due to presence of the rop gene in combination with the pMB1 origin of replication (14), or the chromosomal integrations (Figure 2B, left panel). Although all experiments were performed in the presence of the appropriate antibiotic to avoid loss of the plasmid, all three strains harboring the plasmid-borne reporter genes showed heterogeneous distribution of promoter activity. In contrast, the chromosomal $\mathrm{P}_{\text {luxc }}{ }^{-}$ $m$ Cherry and $\mathrm{P}_{\text {hexA }}$-mCherry reporters both showed a homogeneous distribution at the single-cell level. Only the $\mathrm{P}_{\text {ant }}-m$ Cherry reporter strain displayed a heterogeneous distribution of reporter gene activity. The promoterless negative control (i.e., no promoter present upstream of mCherry) showed no fluorescence, as expected, either when plasmid-borne or when chromosomally integrated.

We calculated the noise values of all the reporter strains in order to determine the degree of heterogeneity (Figure 2B, right panel). The maximum noise level in an apparently homogeneous population was set to 0.38 , as determined for the $\mathrm{P}_{\text {luxc }}$-mCherry chromosomal reporter. The chromosomal promoter activity of $\mathrm{P}_{\text {hexA }}$ with a noise value of 0.25 also displayed a homogeneous distribution of reporter gene activity. For $\mathrm{P}_{\text {ant }}$, a higher noise value of 0.56 was calculated, confirming the heterogeneous distribution of $\mathrm{P}_{\text {ant }}$ activity. In contrast, the fluorescence noise values of all 3 plasmid-borne reporter genes were calculated to be 3- to 5-fold higher compared with that of the chromosomal integrations, which confirmed the high degree of heterogeneity at the single-cell level for all of the plasmid-encoded reporter genes used in this study.

To test if the remaining pPINTmCherry vector backbone had an influence on the promoter activity, we compared the fluorescence intensities and noise values of the $P_{\text {anta }}-m$ Cherry reporter strain with a similar reporter strain, P. luminescens $\mathrm{P}_{\text {anta }}$-mCherry/ $\mathrm{Kan}^{\mathrm{S}}$, in which the plasmid backbone has been removed (see Supplementary Material for detail). Correct loss of the vector backbone was checked by PCR (Figure 3A). Fluorescence intensities and noise levels were comparable for both reporter strains, revealing that the pPINT-mCherry plasmid backbone does not interfere with the respective promoter activity and only needs to be removed if the reporter strain must be kanamycin sensitive (Figure 3B).

To analyze whether the heterogeneous distribution of plasmid-borne promoter activities in P. luminescens was caused by unequal plasmid distribution, we analyzed the fluorescence of $E$. coli and $P$. luminescens harboring a reporter plasmid encoding both $P_{\text {hexA }}{ }^{-}$ $m$ Cherry and $\mathrm{P}_{\text {lac }}$-eyfp fusions. The $E$. coli reporter strain showed a rather homogeneous distribution of the $P_{\text {hexA }}$ and $P_{l a c}$ activities at the single-cell level. In contrast, the $P$. luminescens reporter strain showed a heterogeneous distribution of both promoter activities. However, the two fluorophores were always present within the same cells, or the cells were completely dark (Figure 4). This suggests that the presence or absence of the fluorescent proteins in principle does not at all mark the active state of the corresponding promoter but is probably due to a non-homogeneous distribution of the plasmid among the population.

Recently, another method to perform chromosomal insertions into 
the $P$. luminescens genome has been developed based on a $P$. luminescens endogenous recombinase system that is homologous to lambda-red (15), which is a useful tool for integrating promoter insertions or gene knockouts into the genome using linear DNA fragments. However, since $P$. luminescens does not grow at $37^{\circ} \mathrm{C}$, it is challenging to remove the temperature-sensitive plasmid that carries the recombinase genes after the recombination event.

In summary, we have created an efficient tool for integrating (reporter) genes at a unique site in the $P$. luminescens genome. We showed that plasmids cannot be used to investigate promoter activities at the single-cell level, and thus, the stable integration of promoter-reporter genes into the genome is necessary for studying heterogeneity in $P$. luminescens. Here we used the low-copy, pBR322-derived plasmid pBR-Cherry for single-cell analysis. However, we cannot exclude the possibility that other plasmids known to be highly stable in a broad host range, such as pBBR1 derivatives (16), might also be useful tools in Photorhabdus if high fluorescence readouts are essential when analyzing activities of weak promoters. In principle, plasmid-based reporter systems are preferred as they are timesaving compared with chromosomal integrations. Furthermore, due to their higher copy number, plasmidencoded reporter systems often result in higher signals compared with chromosomally encoded reporter genes. However, we have shown that plasmids seem to be unequally distributed in a $P$. luminescens population, which has also been suggested previously (17). Therefore, plasmid-encoded promoterreporter genes are sufficient in these bacteria only when analyzing reporter gene activities at the population level. The rpmE/g/mS intergenic region that we used as the integration site is also present in the genomes of the other two known Photorhabdus species, $P$. temperata and $P$. asymbiotica, suggesting that the pPINT-mCherry tool might be of use for performing chromosomal integrations in these organisms as well.

\section{Author contributions}

A.G. performed the experiments. R.H. coordinated the experiments. A.G. and R.H. wrote the manuscript.

\section{Acknowledgments}

This work was supported by the Deutsche Forschungsgemeinschaft (SPP 1617, HE-5247/5-1).

\section{Competing interests}

The authors declare no competing interests.

\section{References}

1. Veening, J.-W., W.K. Smits, and O.P. Kuipers. 2008. Bistability, Epigenetics, and Bet-Hedging in Bacteria. Annu. Rev. Microbiol. 62:193-210.

2. Clarke, D.J. 2008. Photorhabdus: a model for the analysis of pathogenicity and mutualism. Cell. Microbiol. 10:2159-2167.

3. Ehlers, R.U. 2003. Entomopathogenic nematodes in the European biocontrol market. Commun. Agric. Appl. Biol. Sci. 68:3-16.

4. Bode, H.B. 2009. Entomopathogenic bacteria as a source of secondary metabolites. Curr. Opin. Chem. Biol. 13:224-230.

5. Akhurst, R.J. 1980. Morphological and functional dimorphism in Xenorhabdus spp, bacteria symbiotically associated with the insect pathogenic nematodes Neoplectana and Heterorhabditis. J. Gen. Microbiol. 121:303-309.

6. Boemare, N.E. and R.J. Akhurst. 1988. Biochemical and physiological characterization of colony form variants in Xenorhabdus spp. (Enterobacteriacea). J. Gen. Microbiol. 134:751-761.

7. Thoma, S. and M. Schobert. 2009. An improved Escherichia coli donor strain for diparental mating. FEMS Microbiol. Lett. 294:127-132.

8. Lassak, J., A.-L. Henche, L. Binnenkade, and K.M. Thormann. 2010. ArcS, the cognate sensor kinase in an atypical Arc system of Shewanella oneidensis MR-1. Appl. Environ. Microbiol. 76:3263-3274.

9. Münch, A., L. Stingl, K. Jung, and R. Heermann. 2008. Photorhabdus luminescens genes induced upon insect infection. BMC Genomics 9:229.

10. Hanahan, D. 1985. In D. Glover (Ed.) DNA Cloning, Volume 1. IRL Press, Ltd., London, U.K.

11. Plener, L., N. Lorenz, M. Reiger, T. Ramalho, U. Gerland, and K. Jung. 2015. The Phosphorylation Flow of the Vibrio harveyi Quorum-Sensing Cascade Determines Levels of Phenotypic Heterogeneity in the Population. J. Bacteriol. 197:1747-1756.

12. Choi, K.-H. and H.P. Schweizer. 2006. mini-Tn7 insertion in bacteria with single attTn7 sites: example Pseudomonas aeruginosa. Nat. Protoc. 1:153-161.
13. Quandt, J. and M.F. Hynes. 1993. Versatile suicide vectors which allow direct selection for gene replacement in Gram-negative bacteria. Gene 127:15-21.

14. Sambrook, J., E.F. Fritsch, and T. Maniatis. 1989. Molecular Cloning: A Laboratory Manual. Cold Spring Harbor Laboratory Press, Cold Spring Harbor, NY.

15. Yin, J., H. Zhu, L. Xia, X. Ding, T. Hoffmann, M. Hoffmann, X. Bian, R. Müller, et al. 2015. A new recombineering system for Photorhabdus and Xenorhabdus. Nucleic Acids Res. 43:e36.

16. Miller, W.G., J.H.J. Leveau, and S.E. Lindow. 2000. Improved gfp and inaZ BroadHost-Range Promoter-Probe Vectors. Mol. Plant Microbe Interact. 13:1243-1250.

17. O'Neill, K.H., D.M. Roche, D.J. Clarke, and B.C.A. Dowds. 2002. The ner gene of Photorhabdus: effects on primary-form-specific phenotypes and outer membrane protein composition. J. Bacteriol. 184:3096-3105.

Received 10 April 2015; accepted 28 May 2015.

Address correspondence to Ralf Heermann, Ludwig-Maximilians-Universität München, Biozentrum, Bereich Mikrobiologie, Martinsried/München, Germany. E-mail: heermann@Imu.de

To purchase reprints of this article, contact: biotechniques@fosterprinting.com 